\title{
Awards of ICCM 2013
}

\section{by the Editors}

\section{Morningside Medal of Mathematics Selection Committee}

The Morningside Medal of Mathematics Selection Committee comprises a panel of world renowned mathematicians and is chaired by Professor Shing-Tung Yau. A nomination committee of around 50 mathematicians from around the world nominates candidates based on their research, qualifications, and curriculum vitae. The Selection Committee reviews these nominations and recommends up to two recipients for the Morningside Gold Medal of Mathematics, up to two recipients for the Morningside Gold Medal of Applied Mathematics, and up to four recipients for the Morningside Silver Medal of Mathematics. The Selection Committee members, with the exception of the committee chair, are all non-Chinese to ensure the independence, impartiality and integrity of the awards decision.

Members of the 2013 Morningside Medal of Mathematics Selection Committee are:

\section{Richard E. Borcherds}

Professor Borcherds is Professor of Mathematics at the University of California at Berkeley. His research interests include Lie algebras, vertex algebras, and automorphic forms. He is best known for his work connecting the theory of finite groups with other areas in mathematics. Professor Borcherds was awarded the Fields Medal in 1998 for his work in algebra and geometry, in particular for his proof of the Moonshine conjecture.

\section{John H. Coates}

Professor Coates is Emeritus Sadleirian Professor of Pure Mathematics at the University of Cambridge. He is a number theorist, with particular research interests in Iwasawa theory and the arithmetic of elliptic curves. He frequently visits Asia, and is currently a Distinguished Professor at the Pohang University of Science and Technology, South Korea. Professor Coates is the first recipient of the ICCM International Cooperation Award in 2004.

\section{Simon Donaldson}

Professor Donaldson is Royal Society Research Professor at Imperial College. His research interests include differential geometry and its connections with differential topology and algebraic geometry. Professor Donaldson is a Fellow of the Royal Society, and a foreign member of the academies of France, Sweden and the United States. He is a recipient of the Fields Medal (1986), the Crafoord Prize in Mathematics (1994), the King Faisal International Prize for Science (2006), and the Shaw Prize in Mathematical Sciences (2009).

\section{Björn Engquist}

Professor Engquist is the Computational and Applied Mathematics Chair Professor at the University of Texas at Austin. His recent work includes homogenization theory, multi-scale methods, and fast algorithms for wave propagation. He is a member of the Royal Swedish

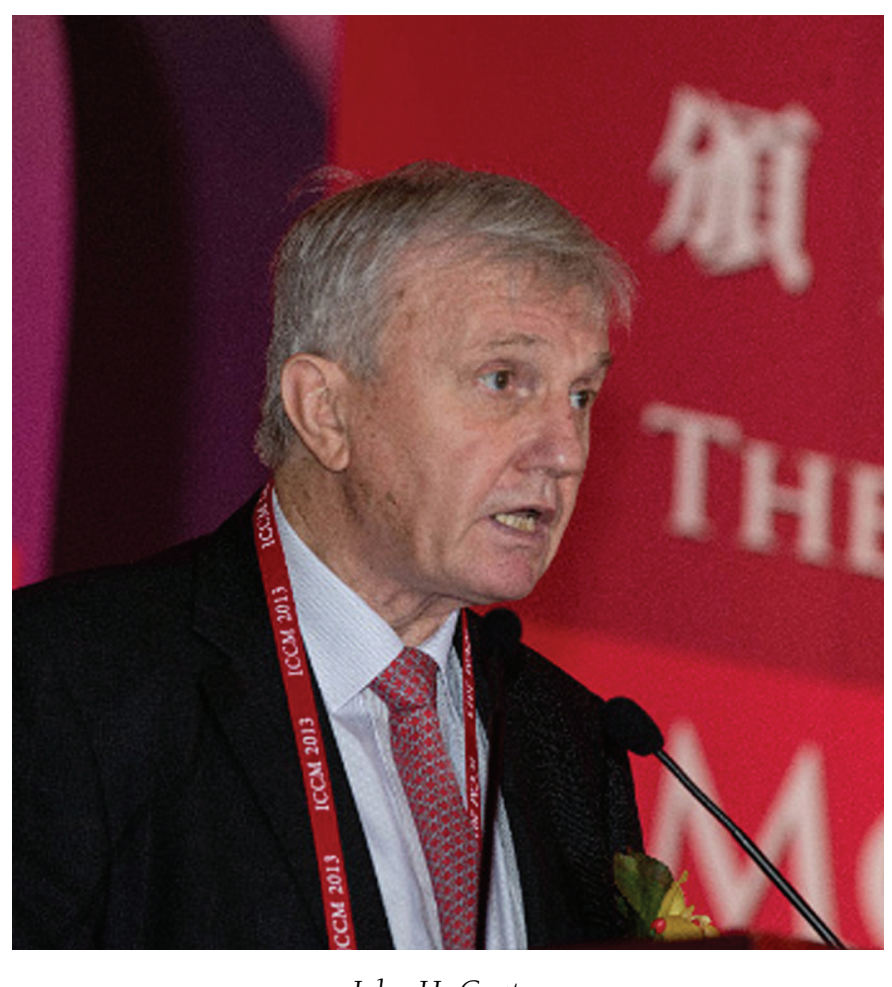

John H. Coates

Academy of Sciences, the Royal Swedish Academy of Engineering Sciences, and the Norwegian Academy of Science and Letters. He was a Guggenheim Fellow (1991), and received the first James H. Wilkinson Prize in Numerical Analysis and Scientific Computing (1982), the Peter Henrici Prize (2011), and the Birkhoff Prize in Applied Mathematics (2012).

\section{Gerd Faltings}

Professor Faltings is a Director of the Max Planck In- 
stitute for Mathematics in Germany. His research interests include number theory and algebraic and arithmetic geometry. Professor Faltings taught at Princeton University and the University of Wuppertal before joining the Max Planck Institute for Mathematics in 1994. Past honors include the Fields Medal (1986), a Guggenheim Fellowship (1988), the Gottfried Wilhelm Leibniz Prize (1996), the Karl Georg Christian von Staudt Prize (2008), and the Heinz Gumin Prize (2010).

\section{James G. Glimm}

Professor Glimm is a Distinguished Professor of Applied Mathematics at the State University of New York at Stony Brook. He has made original contributions in a variety of areas in pure and applied mathematics, including operator algebras, quantum field theory, statistical physics, partial differential equations and scientific computing. His past honors include a Guggenheim Fellowship (1963), the Dannie Heineman Prize for Mathematical Physics (1980), the Leroy P. Steele Prize (1993), and the U.S. National Medal of Science (2002).

\section{Dorian M. Goldfeld}

Professor Goldfeld is a Professor of Mathematics at Columbia University. His primary research interests include number theory and cryptography. Past honors include a Sloan Research Fellowship (1977), the Vaughan Prize (1985), and the Frank Nelson Cole Prize in Number Theory (1987). He is a Fellow of the American Academy of Arts and Sciences, the Editor of Acta Arithmetica and The Ramanujan Journal, and co-founder and board member of SecureRF.

\section{Benedict H. Gross}

Professor Gross is the George Vasmer Leverett Professor of Mathematics at Harvard University and former Dean of Harvard College. He has made several fundamental contributions to mathematics, namely number theory and algebraic geometry. Past honors include a Marshall Scholarship (1972-1974), a MacArthur Fellowship (1986), and the Frank Nelson Cole Prize in Number Theory (1987).

\section{Victor W. Guillemin}

Professor Guillemin is Professor of Mathematics at the Massachusetts Institute of Technology. His research interests are differential geometry and symplectic geometry. He is a Fellow of the American Academy of Arts and Sciences and the U.S. National Academy of Sciences. Past honors include a Guggenheim Fellowship (1988), a Humboldt Research Fellowship (1988), and the Leroy P. Steele Prize for Lifetime Achievement (2003). He is the Editor-in-Chief of the Journal of Symplectic Geometry.

\section{Yuri I. Manin}

Professor Manin is a Professor Emeritus at the Max Planck Institute for Mathematics and Northwestern University. Professor Manin's work includes algebraic geometry, number theory, differential equations, and mathematical physics, and his main research preoccupation of the last decade was quantum cohomology. Past honors include the Moscow Mathematical Society Award (1963), the Lenin Prize (1967), the Dutch Mathematical Society Brouwer Medal (1987), the Frederic Esser Nemmers Prize in Mathematics (1994), the Rolf Schock Prize in Mathematics (1999), the King Faisal International Prize for Science (2002), the Cantor Medal by the German Mathematical Society (2002), and the János Bolyai International Prize by the Hungarian Academy of Sciences (2010).

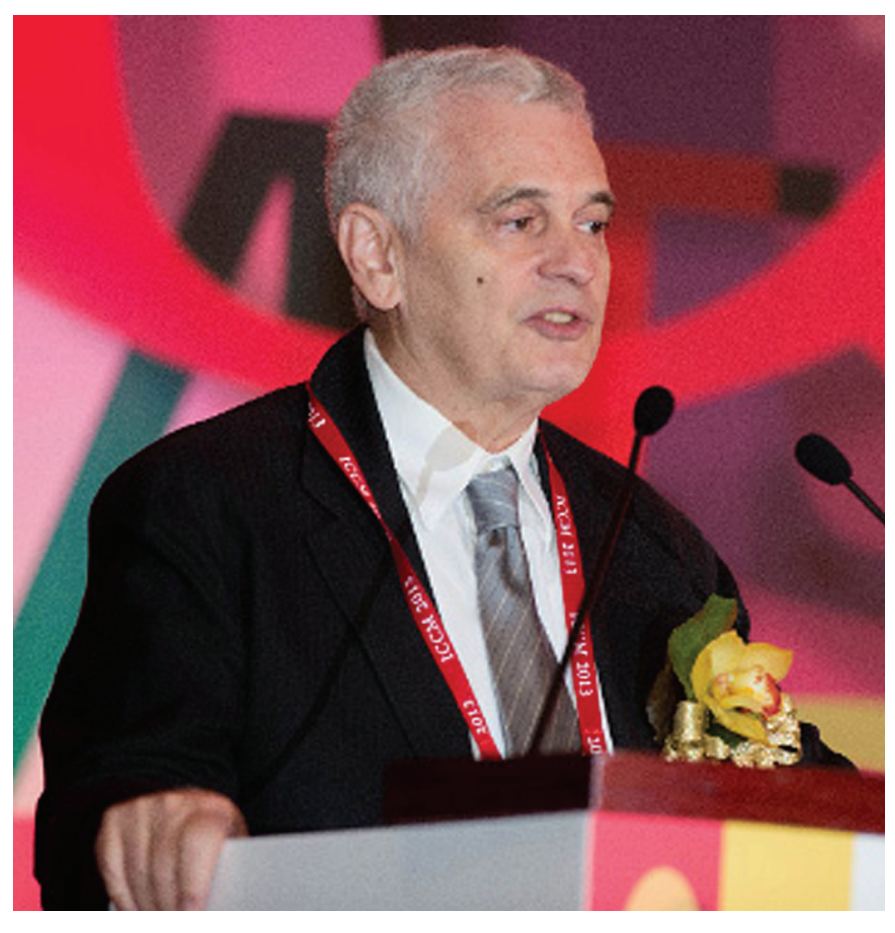

Dorian M. Goldfeld

\section{Stanley J. Osher}

Professor Osher is a Professor of Mathematics at the University of California at Los Angeles, and has joint faculty appointments at the Electrical Engineering and Computer Science Departments. He is also the Director of Special Projects at the University's Institute for Pure and Applied Mathematics. He is the co-inventor and developer of many numerical methods for computational physics and image processing. Past honors include a Sloan Research Fellowship (1972), the NASA Public Service Group Achievement Award (1992), the Computational Mechanics Award by the Japan Society of Mechanical Engineering (2002), the Ralph E. Kleinman Prize (2005), and the Computational and Applied Science Award by the U.S. Association for Computational Mechanics (2007). 


\section{Shing-Tung Yau}

Professor Yau is the William Caspar Graustein Professor of Mathematics at Harvard University, Director of The Institute of Mathematical Sciences at The Chinese University of Hong Kong, and Director of the Morningside Center of Mathematics at the Chinese Academy of Sciences. His research interests include differential geometry, differential equations, and general relativity. $\mathrm{He}$ is a member of several academies including Academia Sinica, the Chinese Academy of Sciences, the Russian Academy of Sciences, and the U.S. National Academy of Sciences. Professor Yau has been one of the key individuals behind the founding of the Morningside Center of Mathematics, the Morningside Medal of Mathematics, and the International Congress of Chinese Mathematicians. Past honors include the Fields Medal (1982), the Crafoord Prize in

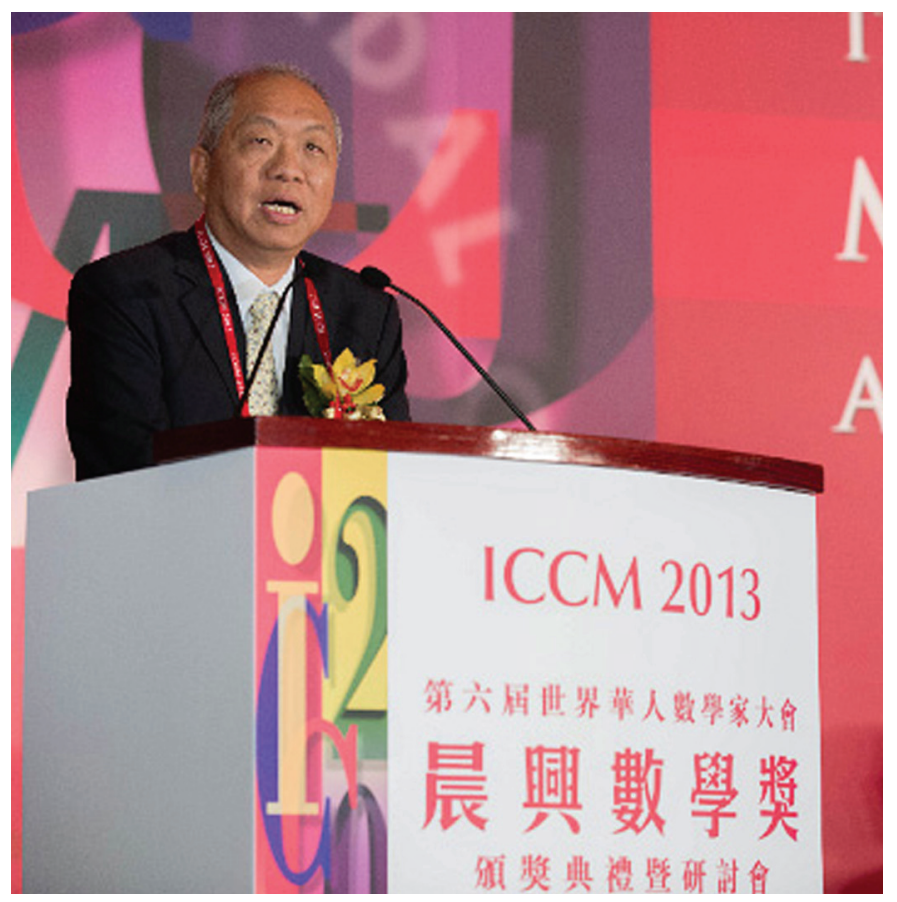

Shing-Tung Yau

Mathematics (1994), the U.S. National Medal of Science (1997), and the Wolf Prize (2010).

\section{Morningside Special Achievement Award in Mathematics}

\section{Yitang Zhang}

Dr. Zhang is awarded the Morningside Special Achievement Award in Mathematics for his proof of the restricted twin prime conjecture. For many years, number theorists have speculated that there are infinitely many twin primes, or pairs of primes with gaps equal to 2 , and this has remained one of the oldest open problems in mathematics. In 2013, Dr. Zhang astounded the mathematical world by showing there exists infinitely many pairs of prime numbers with gaps less than 70,000,000. His paper "Bounded Gaps between Primes" was accepted for publication by the Annals of Mathematics in record time.

Dr. Zhang received his B.S. and M.S. degrees from Peking University, and later his doctorate degree from Purdue University in 1991. Before securing an academic position, he had worked at various jobs before being hired in 1999 by the University of New Hampshire as a substitute lecturer, and a full-time lecturer since 2005. Although Dr. Zhang never held a regular research position and had some hardships in his career, his passion and devotion to mathematics never waned, and in 2013 he marveled the mathematical world with his revelations. Dr. Zhang's perseverance against the odds, and his steadfast pursuit for mathematical truth, is an inspiration to us all.

\section{Citation for Yitang Zhang}

by John H. Coates, Emeritus Sadleirian Professor of

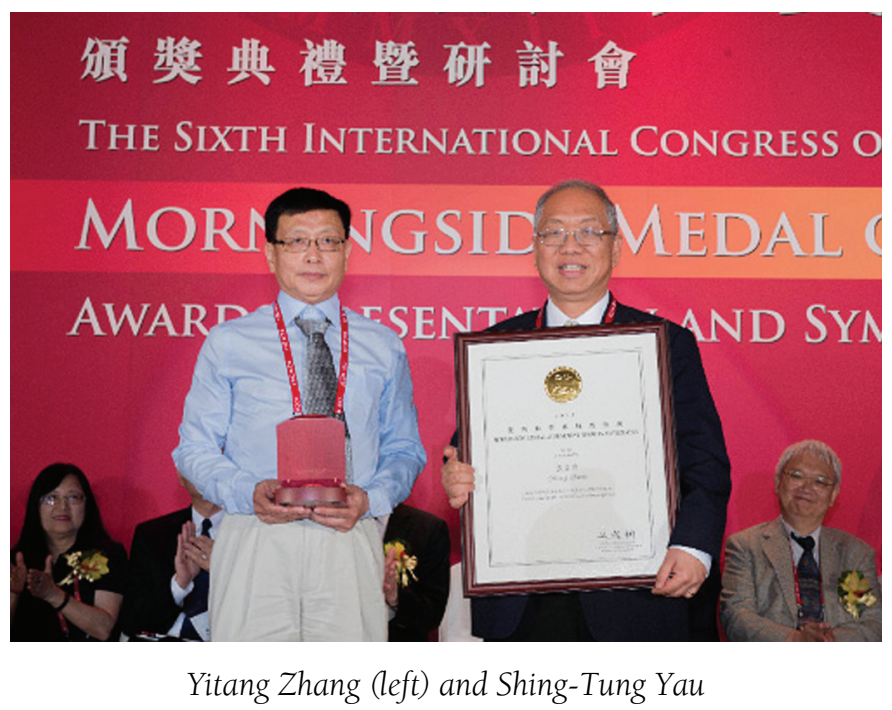

Pure Mathematics, University of Cambridge

The work of Yitang Zhang on the twin prime conjecture breaks entirely new ground, and is unquestionably one of the most surprising and important results in modern number theory.

Prime numbers are the building blocks of arithmetic, and the study of their distribution has always been one of the major themes of research in number theory. The particular question of proving that there are infinitely many primes $p$ such that $p+2$ is also a prime is probably very ancient, although its written history only seems to have started in the 19th century. As our theoretical understanding of some aspects of the distribution of prime numbers grew, great efforts have been made by number theorists from the early 20th century until the present day to attack this so called twin prime conjecture. The best earlier result in its direction was established by the Chinese mathematician Jing-Run Chen in 1966, when he proved that there are infinitely many primes $p$ such that 


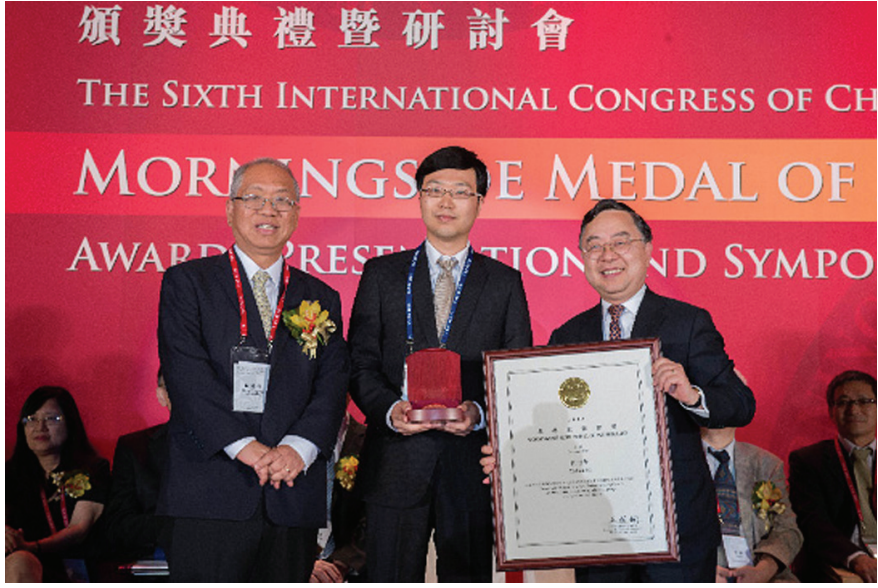

Left to right: Shing-Tung Yau, Xuhua He and Ronnie C. Chan

$p+2$ is either a prime or the product of two primes. It is remarkable that the decisive progress on this problem has now been made by another Chinese mathematician Yitang Zhang. In a manuscript which has greatly surprised the mathematical world, Zhang has proven that there is some fixed integer $c$, less than 70,000,000, such that there are infinitely many primes $p$ with the property that $p+c$ is also a prime. No result of this kind, establishing the existence of infinitely many prime pairs with a constant gap between them, has ever been proven before.

While it is too technical to enter into the detailed mathematical aspects of Zhang's work here, his starting point was the work of Goldston, Pintz, and Yildirim, published in 2009 and 2010, which, very roughly speaking, proved a slightly weaker form of the assertion that there are infinitely many prime pairs which differ by less than the square root of the logarithm of the smaller prime in the pair. This result created very considerable interest, but the leading experts in the field failed to make the all important improvement to get a constant gap between the pairs of primes. Now Zhang has brilliantly succeeded in doing this, by combining these methods of Goldston, Pintz, and Yildirim, with his own original variant of some deep earlier work by Bombieri, Fouvry, Friedlander, and Iwaniec from the 1980's. This is a mathematical achievement of the highest order. Yitang Zhang richly deserves a Morningside Special Achievement Award in Mathematics for this work.

\section{Morningside Medals of Mathematics}

\section{Morningside Gold Medal of Mathematics}

\section{Xuhua He}

Professor He is awarded the 2013 Morningside Gold Medal of Mathematics for his contributions to several fundamental problems in arithmetic algebraic geometry, algebraic groups, and representation theory. He joined The Hong Kong University of Science and Technology (HKUST) in 2008, and is now an Associate Professor of Mathematics. After receiving his Ph.D. in mathematics from the Massachusetts Institute of Technology, he had worked at the Institute for Advanced Study and the State University of New York at Stony Brook before joining HKUST. Professor He's research interests include algebraic group theory, representation theory, combinatorics, algebraic geometry, and arithmetic geometry. Together with his collaborators, Professor $\mathrm{He}$ has solved long-standing open problems on Deligne-Lusztig theory and on Galois cohomology of algebraic groups. His work was reported by Professor Tonny Albert Springer at the International Congress of Mathematicians in 2006, and Professor Claire Voisin at the Bourbaki Seminar in 2011.

\section{Ye Tian}

Professor Tian is awarded the 2013 Morningside Gold Medal of Mathematics for his in-depth and original work in arithmetic algebraic geometry, especially on the congruent number problem. He is Professor at the Morningside Institute of Mathematics and the AMSS at the Chinese Academy of Sciences. He received his B.S. and M.S. degrees in pure mathematics from Sichuan University, and his Ph.D. in mathematics from Columbia University. He then worked at the Institute for Advanced Study and McGill University before joining the Morningside Institute and the AMSS in 2006. Professor Tian's research interests include arithmetic geometry and number theory. Together with his collaborators, Professor Tian solved some long-standing open problems on Birch and Swinnerton-Dyer conjecture and Diophantine equations. He is one of the recipients of the 2007 Morningside Silver Medal of Mathematics.

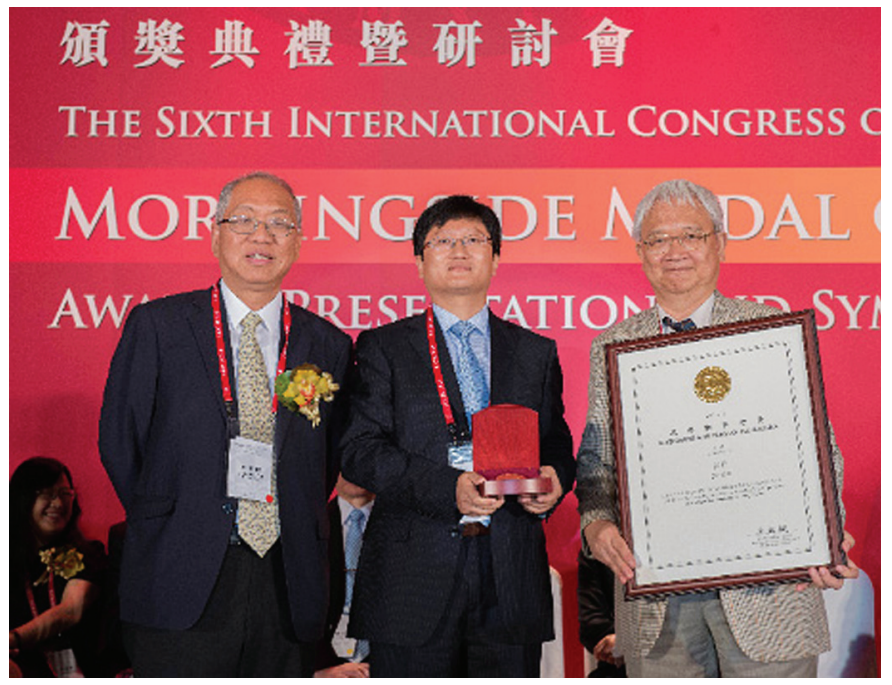

Left to right: Shing-Tung Yau, Ye Tian and Jing Yu 


\section{Morningside Gold Medal of Applied Mathematics}

\section{Xianfeng David Gu}

Professor Gu is awarded the 2013 Morningside Gold Medal of Applied Mathematics for his fundamental contributions to computing conformal structures, computer graphics, medical imaging, and 3-dimensional imaging. He is currently an Associate Professor of Computer Science at the State University of New York at Stony Brook. He received his B.S. in mathematics from Tsinghua University, and his M.S. and Ph.D. degrees from Harvard University under the supervision of Professor Shing-Tung Yau. Professor Gu solved with Professor Yau the fundamental problem of computing conformal structures of surfaces with arbitrary topologies based on Hodge theory. This breakthrough lays the foundation of computational conformal geometry, which Professor Gu applies to many fields in engineering and medicine. He is the winner of the U.S. National Science Foundation CAREER Award (2005), the National Natural Science Foundation of China Overseas Chinese Young Scholars Award (2006), and the recipient of several U.S. National Science Foundation and

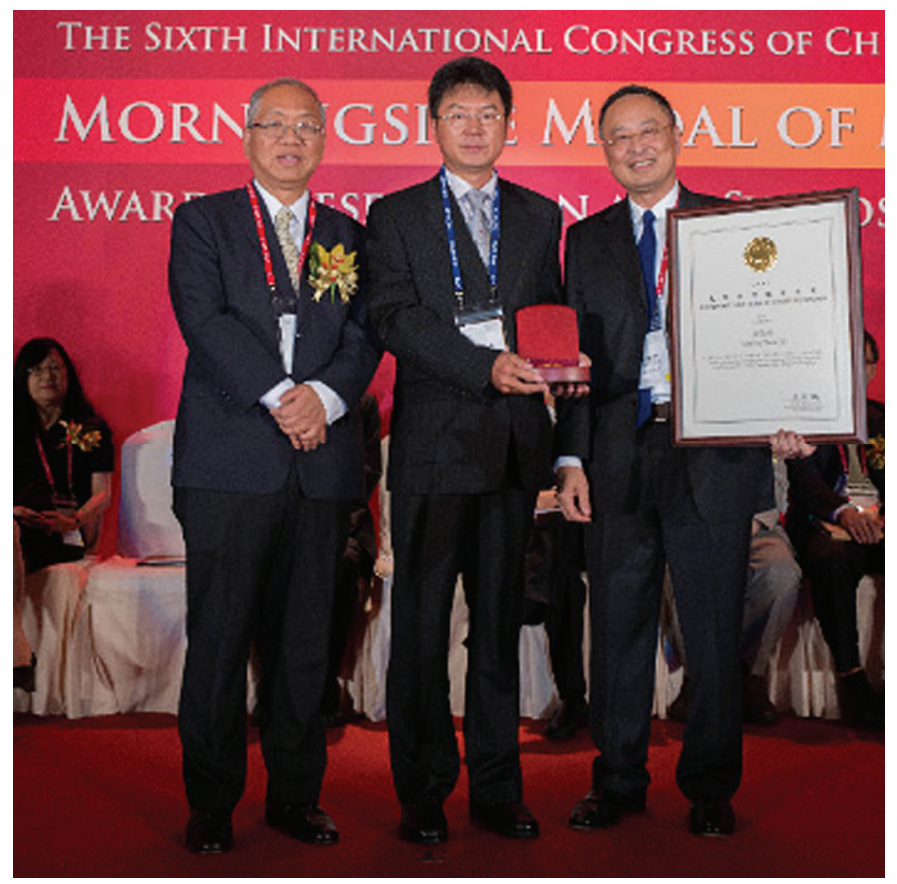

Left to right: Shing-Tung Yau, Xianfeng David Gu and Ronnie C. Chan

U.S. National Institute of Health awards and grants.

\section{Morningside Silver Medal of Mathematics}

\section{Chieh-Yu Chang}

Professor Chang is awarded the 2013 Morningside
Silver Medal of Mathematics for his important contributions to the transcendental number theory in function field. He is an Assistant Professor of Mathematics at National Tsing Hua University in Taiwan. He received his Ph.D. in mathematics from National Tsing Hua University at Taiwan, and completed his postdoctoral research at the National Center for Theoretical Sciences of Taiwan and National Central University. Professor Chang's research interest revolves around the transcendence theory over function fields in positive characteristic, which originates from the foundations that Professor Jing Yu established in the 1980s and 1990s. In recent years, Professor Chang's work is focused on characteristic $p$ multiple zeta values, their transcendence theory and associated arithmetic/ geometric properties.

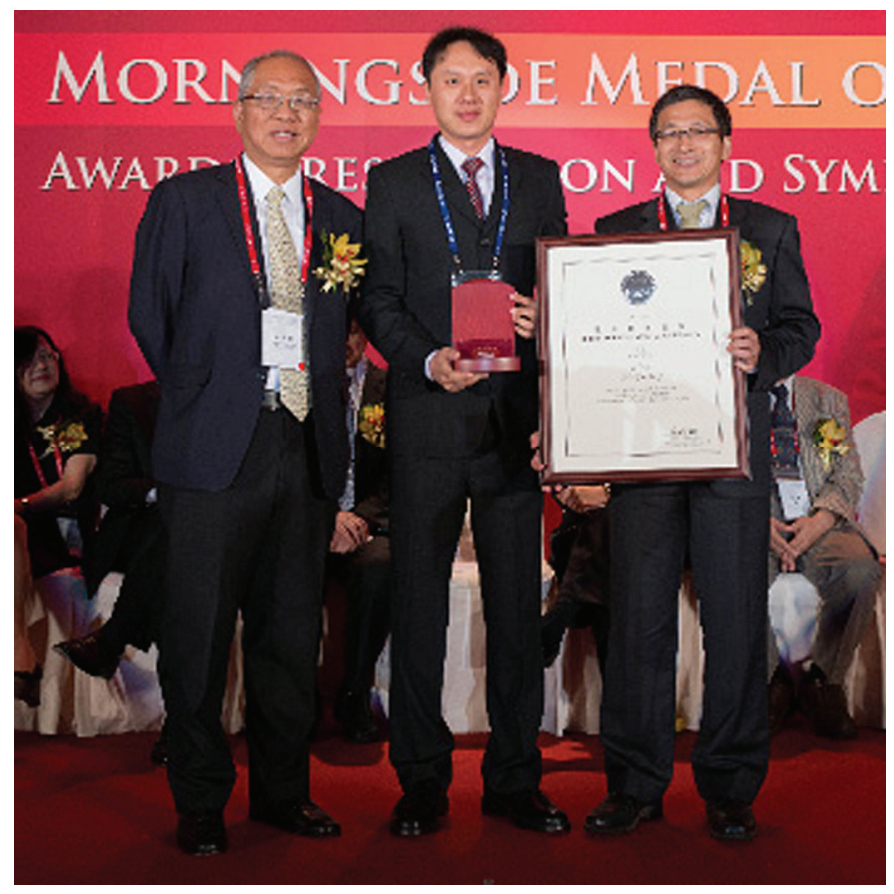

Left to right: Shing-Tung Yau, Chieh-Yu Chang and Shou-Wu Zhang

\section{Xiaoqing $L i$}

Professor Li is awarded the 2013 Morningside Silver Medal of Mathematics for her important research on the analytic properties of automorphic L-functions and their applications. She is an Associate Professor of Mathematics at the State University of New York at Buffalo. She received her Ph.D. in mathematics from Rutgers University under the supervision of Professor Henryk Iwaniec, and received her postdoctoral training at Columbia University for two years. Professor Li's research interests include analytic number theory, automorphic forms and L-functions. Her work has contributed to one of the most important problems in number theory, the Lindeloff hypothesis, which is to prove the sharp upper bound of 


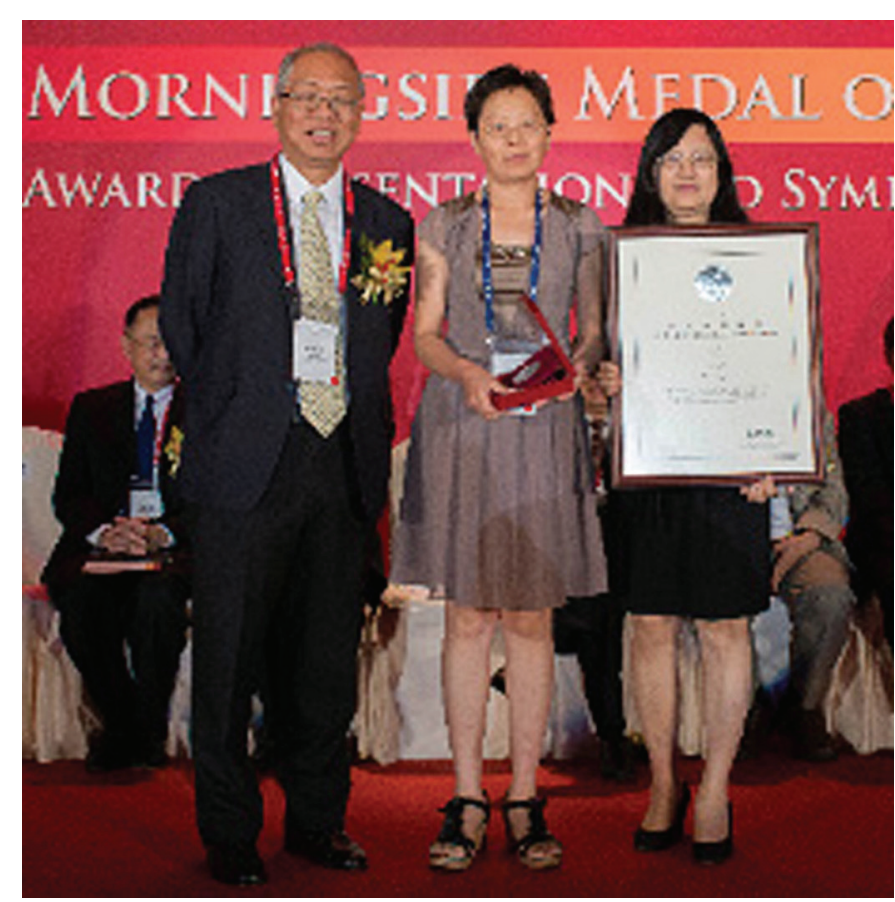

Left to right: Shing-Tung Yau, Xiaoqing Li and Yan-Hwa Wu

L-functions on the one half line.

\section{Hao Xu}

Dr. Xu is awarded the 2013 Morningside Silver Medal of Mathematics for his significant contributions to the intersection theory on the moduli of curves and other related moduli spaces. He received his Ph.D. in mathematics from Zhejiang University under the supervision of Professor Kefeng Liu. He is currently a Research Fellow at Harvard University. Dr. Xu works on intersection theory on moduli spaces of curves and mathematical problems arising in deformation quantization, by using tools from integrable systems, Kähler geometry, Bergman kernels, Feynman diagrams and spectral graph theory. In 2007, he received the Zhong Jiaqing Mathematics Award from the Chinese Mathematical Society, and the New World Mathematics Award Ph.D. Thesis Gold Award at the International Congress of Chinese Mathematicians.

\section{Tai-Peng Tsai}

Professor Tsai is awarded the 2013 Morningside Silver Medal of Mathematics for his contributions to the dynamics of nonlinear Schrödinger equations and the regularity and singularity of the incompressible $\mathrm{Na}$ vier-Stokes equations. He is Professor of Mathematics at the University of British Columbia, and had been a Courant Instructor at New York University from 1998 to 2001, and worked at the Institute for Advanced Study from 2001 to 2002. Professor Tsai received his B.S. in mathematics from National Taiwan University and his Ph.D. from the University of Minnesota. His primary research interests include asymptotics and singularities of solu-

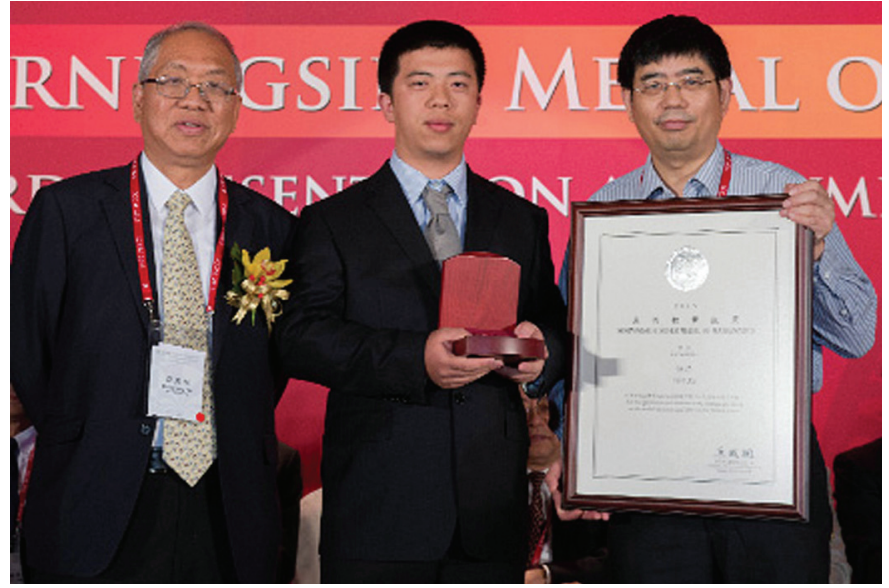

Left to right: Shing-Tung Yau, Hao Xu and Jie Xiao

tions of partial differential equations of fluid and quantum mechanics. In 2006, he received the André Aisenstadt Prize from the Centre de Recherches Mathématiques in Montreal.

\section{ICCM International Cooperation Awards}

The ICCM International Cooperation Award is awarded to an individual who has made significant contributions to the development of mathematics in mainland China, Hong Kong, and Taiwan through collaboration, teaching, and supporting Chinese mathematicians. The award was established in 2004 and is presented every three years at the International Congress of Chinese Mathematicians (ICCM).

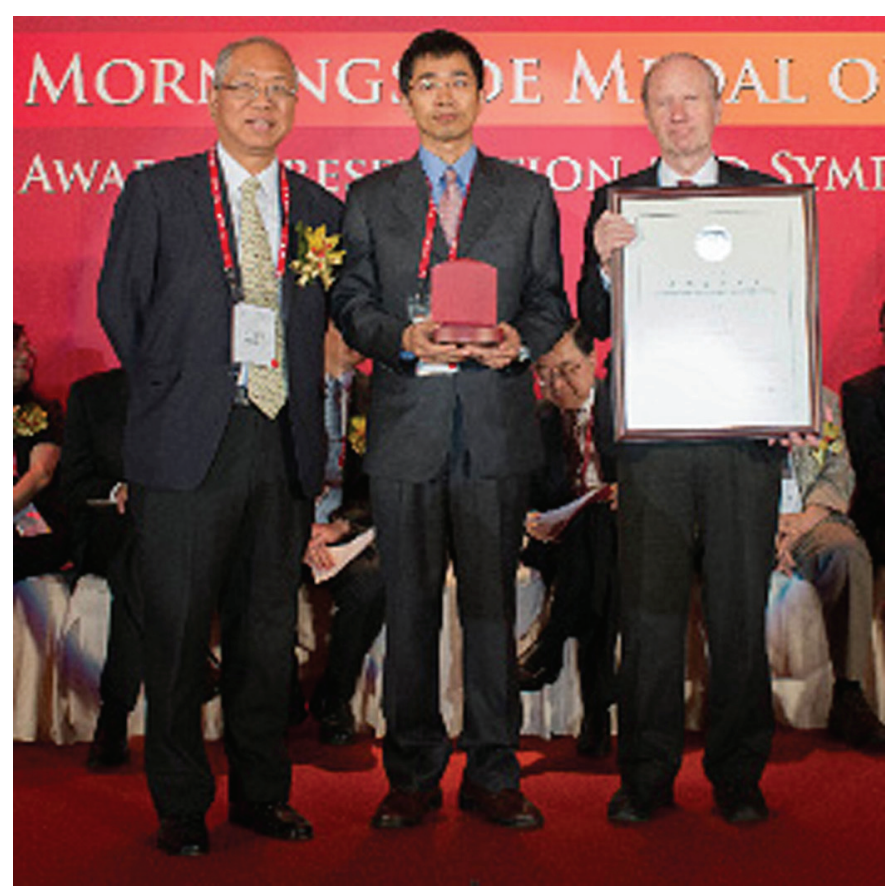

Left to right: Shing-Tung Yau, Tai-Peng Tsai and Björn Engquist 
This year, the Selection Committee of the ICCM International Cooperation Award consists of six members, including Professor Shing-Tung Yau, President of ICCM (chair), Professor Shiu-Yuen Cheng of The Hong Kong University of Science and Technology, Professor Chang-Shou Lin of National Taiwan University, Professor Kefeng Liu of the University of California at Los Angeles and Zhejiang University, Professor Lo Yang of the Chinese Academy of Sciences, and Professor Horng-Tzer Yau of Harvard University.

\section{ICCM International Cooperation Award}

Jean-Pierre Serre

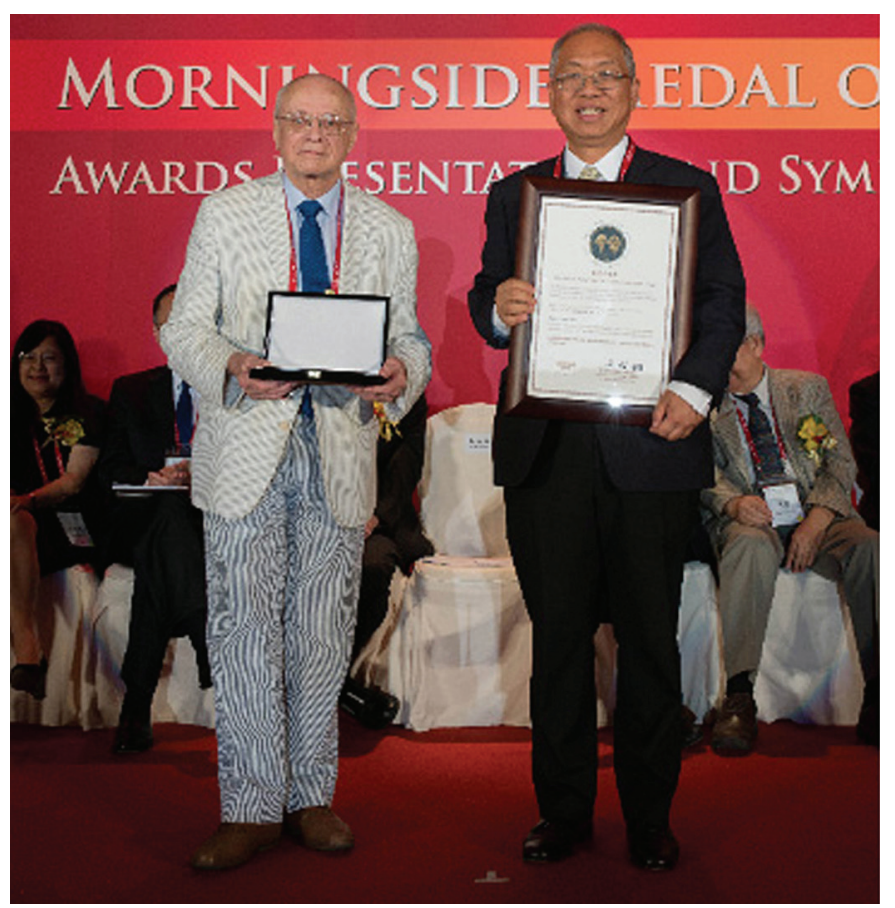

Jean-Pierre Serre (left) and Shing-Tung Yau

Professor Serre is awarded the 2013 ICCM International Cooperation Award for his great achievements and foundational contributions in number theory, algebraic geometry, and algebraic topology, and also for his efforts and support to the Chinese mathematical community. He received his Ph.D. from the Sorbonne in 1951, and from 1956 onwards he was the Chair in Algebra and Geometry at the Collège de France until he retired in 1994 and became an Honorary Professor. As an octogenarian, Professor Serre has been visiting Taiwan biannually since 2009, offering a series of inspiring lectures at the National Center for Theoretical Sciences of Taiwan on his cutting edge research in number theory and arithmetic geometry, and has greatly impacted the Taiwanese mathematical community.

Professor Serre has made foundational contributions in algebraic topology, algebraic geometry, and number theory. In his 1951 thesis, he applied the now-called Leray-Serre spectral sequence to establish connections between the homology and homotopy groups of a space, and computed the homotopy groups of spheres. In 1955 he introduced algebraic coherent sheaves on varieties, extending cohomology theory to algebraic geometry. One year later, he introduced the GAGA theory, connecting analytic geometry and algebraic geometry. Together with John Tate, Professor Serre shaped the landscape of modern algebraic number theory, and introduced and developed algebraic K-theory, Galois cohomology and $\ell$-adic Galois representations.

Professor Serre has been elected to many national academies, in particular the academies of France, Sweden, United States, and the Netherlands. He has received numerous awards, including the Fields Medal in 1954 (the youngest recipient ever), the Prix Gaston Julia in 1970, the Balzan Prize in 1985, the Leroy P. Steele Prize for Mathematical Exposition in 1995, the Wolf Prize in 2000, and the first Abel Prize in 2003. Professor Serre has been awarded honorary degrees from 13 universities, and has been made a Commander Legion d'Honneur and High Officer Ordre National du Merite by the French government.

\section{Chern Prizes}

The Chern Prize was established in 2001 in honor of Professor Shiing-Shen Chern, a mathematician, an educator, and one of the greatest geometers of the 20 century. Presented every three years at the International Congress of Chinese Mathematicians (ICCM), the Chern Prize is awarded to mathematicians of Chinese decent who have made exceptional contributions to mathematical research or to public service activities in support of mathematics.

This year, the Selection Committee of the Chern Prize consists of six members, including Professor Shing-Tung Yau, President of ICCM (chair), Professor Shiu-Yuen Cheng of The Hong Kong University of Science and Technology, Professor Chang-Shou Lin of National Taiwan University, Professor Kefeng Liu of the University of California at Los Angeles and Zhejiang University, Professor Lo Yang of the Chinese Academy of Sciences, and Professor Horng-Tzer Yau of Harvard University.

\section{Chern Prize}

\section{Si-Chen Lee}

Professor Lee is awarded the 2013 Chern Prize in recognition of his efforts and contribution to upgrade the research of mathematics in Taiwan, and with his vision and determination, fully endorsing the establishment of a first-class mathematical community within National Taiwan University. He received his Ph.D. in electrical engineering from Stanford University in 1981. His research 
has led to the innovative development of ledge-type heterojunction bipolar transistor (HBT) in 1985, which has become the norm in manufacturing HBT power amplifiers, dominating the cell phone production in billions of units every year. Professor Lee is an Institute of Electrical and Electronics Engineers (IEEE) Fellow. He received many accolades, including the Dr. Sun Yat-Sen Academic Award (1987), five consecutive Outstanding Research Awards by the National Science Council of Taiwan (1986-1996), the IEEE Third Millennium Medal (2000), the Medal of Electrical Engineering from the Association of Chinese Electrical Engineers (2002), and the 47th Academic Award (Engineering and Applied Science) by the Ministry of Education of Taiwan (2003).

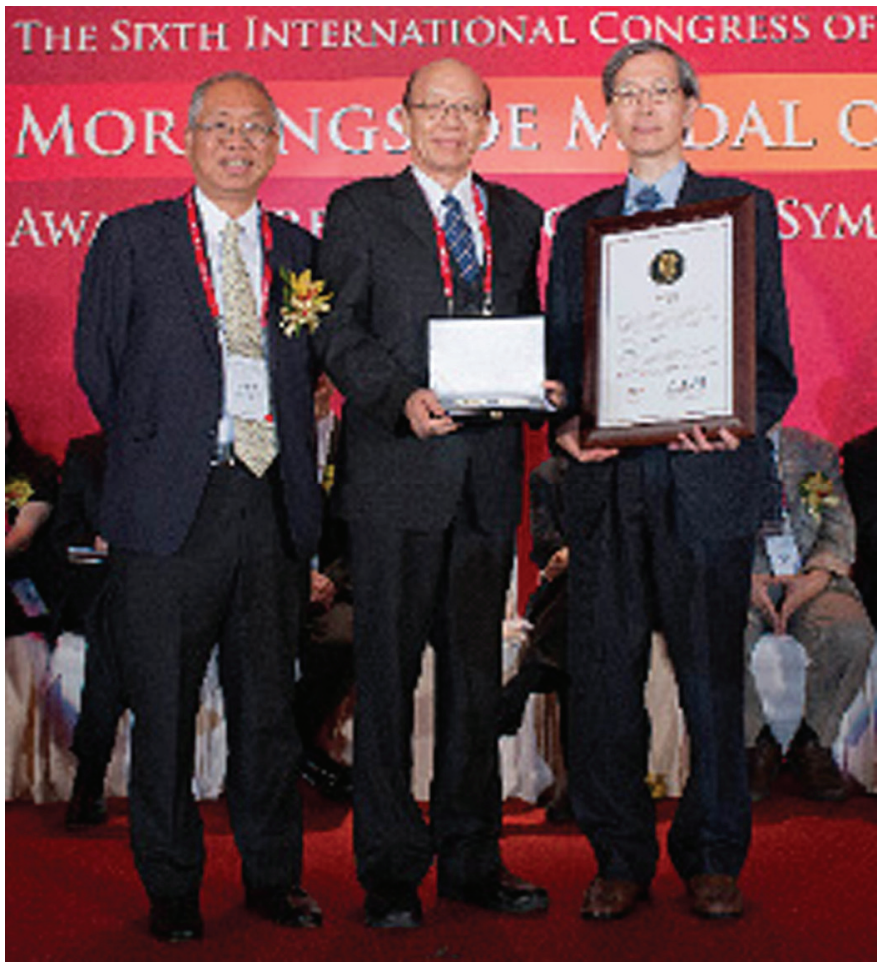

Left to right: Shing-Tung Yau, Si-Chen Lee and Ching-Li Chai

\section{Bong Lian}

Professor Lian is awarded the 2013 Chern Prize for his fundamental contributions to the investigation of Calabi-Yau mirror symmetry and representation theory of vertex operator algebras. He is a Professor of Mathematics at Brandeis University. He received his B.A. from the University of Toronto, and his Ph.D. from Yale University. His research areas are representation theory, Calabi-Yau geometry, and string theory. Among his achievements include his thesis on semi-infinite cohomology theory, which has become a primary tool in the representation theory of the Virasoro algebra. In 1996, he worked with Kefeng Liu and Shing-Tung Yau to solve the outstanding mirror conjecture that is fundamental in enumerative geometry and string theory. This work was also done independently by A. Givental. In 2010, Professor Lian worked with Ruifang Song and Shing-Tung Yau, and jointly developed a new approach, called tautological systems, to study the Riemann-Hilbert problem for period integrals. Recently, on a joint research work with Spencer Bloch, An Huang, Vasudevan Srinivas, Shing-Tung Yau and Xinwen Zhu, Professor Lian solved the completeness problem for tautological systems of period integrals in a number of important cases. He was a Sloan Dissertation Fellow (1990) and a Guggenheim Fellow (2003).

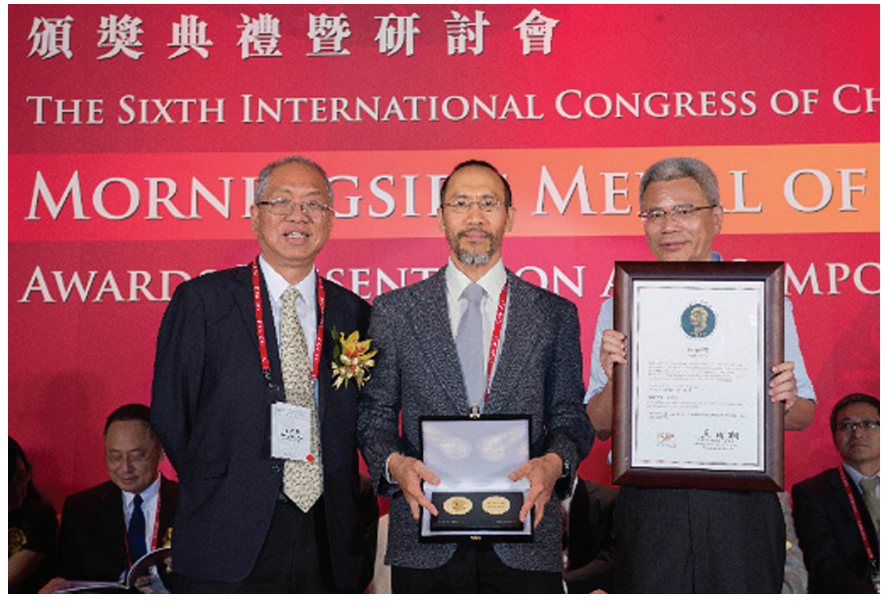

Left to right: Shing-Tung Yau, Bong Lian and Chang-Shou Lin

\section{Morningside Mentor Award in Mathematics}

\section{Chengbiao Pan}

Professor Pan is awarded the Morningside Mentor Award in Mathematics for his tireless efforts in training first class mathematicians. The Morningside Mentor Award in Mathematics is created to recognize the impact of mathematicians who invest in students' well-being through teaching, and in so doing, they pass onto their students a lifelong desire to learn, and inspire them to pursue their goals in mathematics and research work. Professor Pan is not only an inspiration to fellow mathematicians, academic professionals and students, but also a catalyst for positive change, growth, and innovation in the field of mathematics education.

Professor Pan was a Professor at the College of Science at China Agricultural University since 1986, and had held an adjunct position at the Department of Mathematics at Peking University since 1977, until his retirement in 2008. Professor Pan has devoted his work to the field of mathematics, particularly the teaching and research of analytic number theory, and has co-authored numerous academic works in Chinese including Goldbach Conjecture, Algebraic Number Theory, Elementary Num- 
ber Theory, Condensed Number Theory, and Guide to Modular Forms. Besides his research work, Professor Pan was the former editor of Acta Mathematica Sinica and Advances in Mathematics, and a former committee member of the Chinese Mathematical Olympiad. He is a dedicated teacher and mentor to many aspiring mathe-

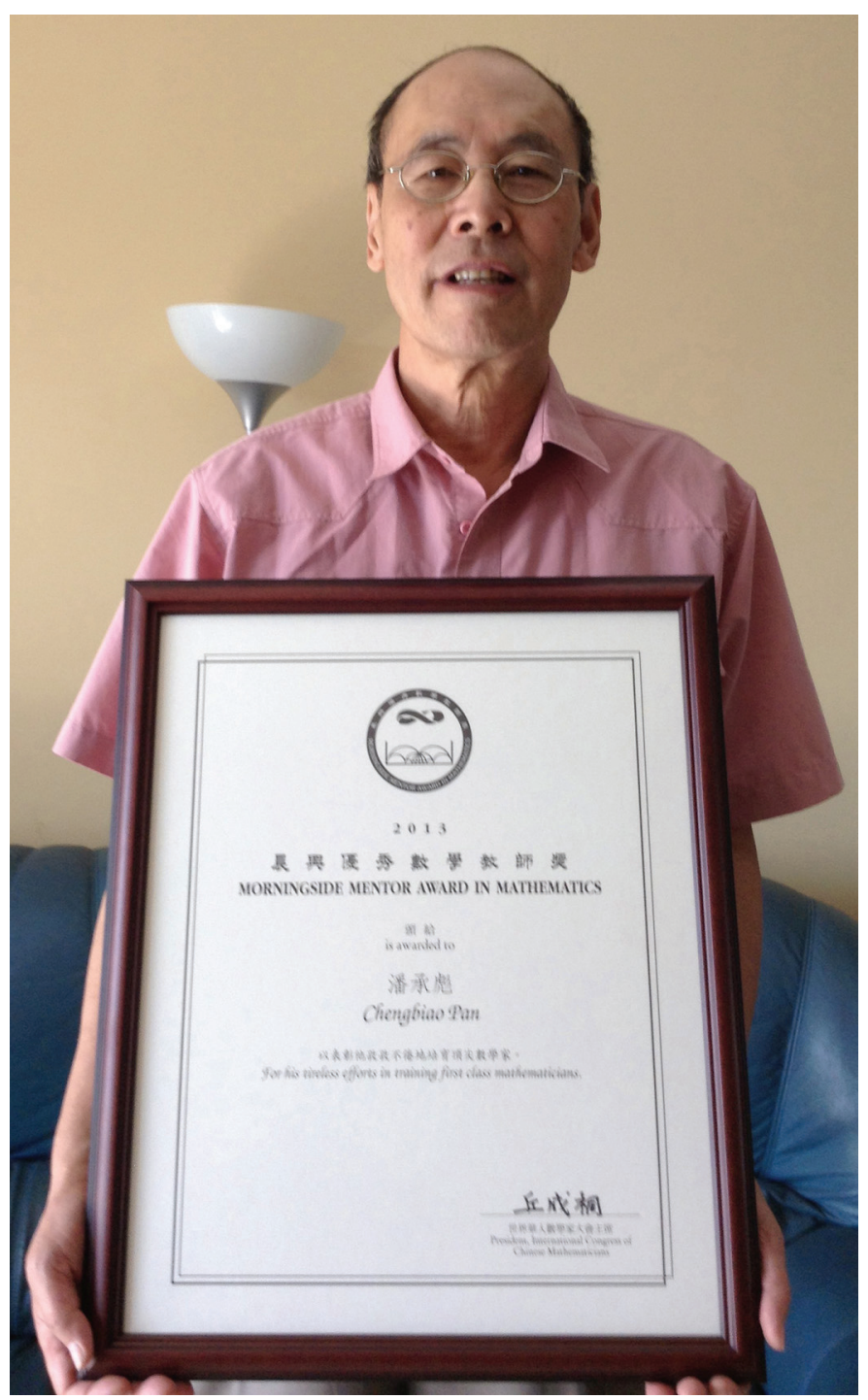

Chengbiao Pan

maticians. Dr. Yitang Zhang of the University of New Hampshire and recipient of the 2013 Morningside Special Achievement Award in Mathematics, Professor Chaohua Jia of the Chinese Academy of Sciences, and Professor Wenzhi Luo of The Ohio State University, to name a few, have all benefited from his tutelage. 